hep-ph/0008144

August 2000

\title{
Neutrino Flavor Oscillations without Flavor Mixing Angles
}

\author{
Keith R. Dienes and Ina Sarcevic' \\ Department of Physics, University of Arizona, Tucson, AZ 85721 USA
}

\begin{abstract}
We demonstrate that sizable neutrino flavor oscillations can be generated in a model with large extra spacetime dimensions even if the physics on the brane is flavor-diagonal, the bulk neutrino theory is flavor-neutral, and the brane/bulk couplings are flavor-blind. This is thus a compact model for addressing neutrino flavor oscillations in higher dimensions. We also discuss several phenomenological aspects of the "bulk-mediated" neutrino oscillations inherent in this model, and show that this model contains some potentially important new phenomenological features in the limit of large brane/bulk coupling.
\end{abstract}

*E-mail address: dienes@physics.arizona.edu

${ }^{\dagger}$ E-mail address: ina@physics.arizona.edu 
Over the past few years, experimental evidence that neutrinos have mass and undergo oscillations has been pointing towards the existence of new physics beyond the Standard Model. Recent SuperK data on solar neutrinos [1] and atmospheric neutrinos [2], combined with the LSND accelerator measurements [3], has provided a new challenge for our understanding of the lepton sector of the Standard Model.

In some sense, the observation of lepton mixing restores a parallel structure between the quark and lepton sectors of the Standard Model. However, although the quark sector of the Standard Model is characterized by relatively small flavor mixing angles, recent data suggest that the lepton sector experiences large flavor mixings. Explaining the origin of these large mixings thus becomes a theoretical challenge. One important observation in this regard is the fact that right-handed quarks carry both color and electromagnetic charge, while right-handed neutrinos are neutral under all Standard-Model gauge symmetries. Thus, it is plausible that right-handed neutrinos have completely different origins than their quark counterparts.

One interesting possibility [4, 5] is that the right-handed neutrino may be realized as a closed- (rather than open-) string state, and hence may propagate in the higher-dimensional bulk corresponding to a large extra spacetime dimension. The possibility of having large extra spacetime dimensions has been proposed [6] as a method of lowering the fundamental high-energy scales of physics such as the GUT, Planck, and string scales. Allowing the right-handed neutrino to feel these extra spacetime dimensions would have profound consequences for neutrino physics; indeed, it might then be possible to use neutrinos as a method of probing the physics of large extra dimensions. Various phenomenological aspects of higher-dimensional neutrino physics have been explored in Refs. [曰, 5, 7].

One key issue concerns the embedding of flavor into such higher-dimensional scenarios. All flavor models in the literature thus far introduce one bulk neutrino for each of the three brane neutrinos, thereby extending flavor into the bulk. However, in such cases, the brane/bulk couplings become arbitrary $3 \times 3$ mixing matrices whose parameters are undetermined. Moreover, the three bulk neutrinos can in principle correspond to different extra spacetime dimensions with different radii. Thus, one obtains a scenario with many undetermined parameters governing neutrino masses and mixing angles.

By contrast, we shall here introduce a "compact" model in which only one bulk neutrino is required. In other words, we shall consider flavor to be a property internal to the Standard Model, one which is restricted to the brane and which therefore does not extend into the bulk. Furthermore we shall assume flavor-blind couplings between our brane and bulk fields. Thus, in this model, only the physics directly on the brane is flavor-sensitive. Moreover, for simplicity we shall also assume that our brane theory is completely flavor-diagonal. Despite these facts, we shall see that neutrino flavor oscillations can still arise.

We begin by briefly describing the model. On the brane, we introduce three lefthanded neutrinos $\nu_{i}(i=1,2,3)$; these are our flavor eigenstates. We assume that 
these left-handed neutrinos have corresponding Majorana masses $m_{i}$ on the brane, and we shall take the $m_{i}$ to be unequal. It is in this way that we shall distinguish between the different flavors on the brane. Note that for our purposes, we shall simply consider these Majorana masses to be arbitrary input parameters, and we shall not speculate on their origins or sizes. Despite the presence of these masses, however, we shall not introduce any explicit flavor mixings between the left-handed neutrinos on the brane. Thus, our theory on the brane will be completely flavor-diagonal.

Our bulk theory will be identical to that considered in Ref. [4]. Specifically, we shall consider a single five-dimensional Dirac fermion $\Psi$, which in the Weyl basis can be decomposed into two two-component spinors: $\Psi=\left(\psi_{1}, \bar{\psi}_{2}\right)^{T}$. This Dirac fermion does not carry any flavor indices, and is therefore completely flavor-neutral. We impose the orbifold relations $\psi_{1,2}(-y)= \pm \psi_{1,2}(y)$ where $y$ is the coordinate of the fifth dimension. In order to have a diagonal mass matrix, we also introduce the linear combinations $N^{(n)} \equiv\left(\psi_{1}^{(n)}+\psi_{2}^{(n)}\right) / \sqrt{2}$ and $M^{(n)} \equiv\left(\psi_{1}^{(n)}-\psi_{2}^{(n)}\right) / \sqrt{2}$ for all $n>0$. Assuming that the brane is located at the orbifold fixed point $y=0$, we see that $\psi_{2}$ vanishes on the brane. The most natural brane/bulk coupling is therefore simply between $\nu_{i}$ and $\psi_{1}$. Although in principle each left-handed neutrino $\nu_{i}$ on the brane can have a different coupling to $\psi_{1}$, we shall consider a simple model in which this brane/bulk coupling, labeled by $\hat{m}$, is completely flavor-universal.

Given these assumptions, our Lagrangian has the form

$$
\begin{aligned}
\mathcal{L}_{\text {brane }} & =\int d^{4} x \sum_{i=1}^{3}\left\{\bar{\nu}_{i} i \bar{\sigma}^{\mu} D_{\mu} \nu_{i}+m_{i}\left(\nu_{i} \nu_{i}+\text { h.c. }\right)\right\} \\
\mathcal{L}_{\text {bulk }} & =\int d^{4} x d y M_{s}\left\{\bar{\psi}_{1} i \bar{\sigma}^{\mu} \partial_{\mu} \psi_{1}+\bar{\psi}_{2} i \bar{\sigma}^{\mu} \partial_{\mu} \psi_{2}\right\} \\
\mathcal{L}_{\text {coupling }} & =\int d^{4} x \sum_{i=1}^{3}\left(\left.\hat{m} \nu_{i} \psi_{1}\right|_{y=0}+\text { h.c. }\right) .
\end{aligned}
$$

Here $M_{s}$ is the mass scale of the higher-dimensional fundamental theory. By compactifying the Lagrangian (11) down to four dimensions we obtain

$$
\begin{aligned}
\mathcal{L}=\int d^{4} x & \left\{\sum_{i=1}^{3} \bar{\nu}_{i} i \bar{\sigma}^{\mu} D_{\mu} \nu_{i}+\bar{\psi}_{1}^{(0)} i \bar{\sigma}^{\mu} \partial_{\mu} \psi_{1}^{(0)}+\sum_{n=1}^{\infty}\left(\bar{N}^{(n)} i \bar{\sigma}^{\mu} \partial_{\mu} N^{(n)}+\bar{M}^{(n)} i \bar{\sigma}^{\mu} \partial_{\mu} M^{(n)}\right)\right. \\
+ & \left\{\sum_{i=1}^{3} m_{i} \nu_{i} \nu_{i}+\frac{1}{2} \sum_{n=1}^{\infty}\left[\left(\frac{n}{R}\right) N^{(n)} N^{(n)}-\left(\frac{n}{R}\right) M^{(n)} M^{(n)}\right]\right. \\
+ & \left.\left.m \sum_{i=1}^{3} \nu_{i}\left(\psi_{1}^{(0)}+\sum_{n=1}^{\infty} N^{(n)}+\sum_{n=1}^{\infty} M^{(n)}\right)+\text { h.c. }\right\}\right\}
\end{aligned}
$$

where $m \equiv \hat{m} / \sqrt{2 \pi M_{s} R}$ is the volume-suppressed brane/bulk coupling resulting from the rescaling of the individual $\psi_{1}^{(0)}, N^{(n)}$, and $M^{(n)}$ Kaluza-Klein modes.

Given the Lagrangian (2), we see that the Standard-Model flavor-eigenstate 
neutrinos $\nu_{i}$ will mix with the entire tower of Kaluza-Klein states of the higherdimensional $\Psi$ field, even though they do not mix directly with each other. Defining

$$
\mathcal{N}^{T} \equiv\left(\nu_{1}, \nu_{2}, \nu_{3}, \psi_{1}^{(0)}, N^{(1)}, M^{(1)}, N^{(2)}, M^{(2)}, \ldots\right)
$$

we see that the mass terms in the Lagrangian (2) take the form $\frac{1}{2}\left(\mathcal{N}^{T} \mathcal{M N}+\right.$ h.c. $)$ where $\mathcal{M}$ takes the symmetric form

$$
\mathcal{M}=\left(\begin{array}{ccccccccc}
m_{1} & 0 & 0 & m & m & m & m & m & \ldots \\
0 & m_{2} & 0 & m & m & m & m & m & \ldots \\
0 & 0 & m_{3} & m & m & m & m & m & \ldots \\
m & m & m & 0 & 0 & 0 & 0 & 0 & \ldots \\
m & m & m & 0 & 1 / R & 0 & 0 & 0 & \ldots \\
m & m & m & 0 & 0 & -1 / R & 0 & 0 & \ldots \\
m & m & m & 0 & 0 & 0 & 2 / R & 0 & \ldots \\
m & m & m & 0 & 0 & 0 & 0 & -2 / R & \ldots \\
\vdots & \vdots & \vdots & \vdots & \vdots & \vdots & \vdots & \vdots & \ddots
\end{array}\right) .
$$

In this model, the bulk theory is flavor-neutral and the brane/bulk couplings are flavor-blind. Moreover, the theory on the brane is flavor-diagonal. Nevertheless, it is immediately apparent the three brane neutrinos will undergo flavor oscillations as a result of their indirect mixings with the bulk Kaluza-Klein neutrinos. In order to demonstrate this explicitly, we can determine the eigenvalues and eigenvectors of this mass matrix. It turns out that the eigenvalues $\lambda$ of the matrix (4) are given exactly as the solutions to the transcendental equation

$$
\tan \pi \lambda R=\pi m^{2} R \sum_{i=1}^{3} \frac{1}{\lambda-m_{i}} .
$$

For each solution $\lambda$ to (5), the corresponding mass eigenstate $\left|\tilde{\nu}_{\lambda}\right\rangle$ is then exactly given by

$$
\begin{aligned}
\left|\tilde{\nu}_{\lambda}\right\rangle=\frac{1}{\sqrt{N_{\lambda}}} & {\left[\left(\sum_{j=1}^{3} \frac{m}{\lambda-m_{j}}\right)^{-1} \sum_{i=1}^{3} \frac{\lambda}{\lambda-m_{i}}\left|\nu_{i}\right\rangle+\left|\psi_{1}^{(0)}\right\rangle\right.} \\
& \left.+\sum_{k=1}^{\infty} \frac{\lambda}{\lambda-k / R}\left|N^{(k)}\right\rangle+\sum_{k=1}^{\infty} \frac{\lambda}{\lambda+k / R}\left|M^{(k)}\right\rangle\right]
\end{aligned}
$$

where $N_{\lambda}$ is an overall normalization constant.

Note that the different flavor eigenstates $\left|\nu_{i}\right\rangle$ consist of different linear combinations of the different mass eigenstates $\left|\tilde{\nu}_{\lambda}\right\rangle$ for each $\lambda$. Thus, the different flavor eigenstates will experience a relative oscillation with each other. This oscillation is entirely "bulk-mediated" in the sense that there are no explicit flavor mixings on the brane; it is the presence of the higher-dimensional bulk which is completely responsible for inducing the flavor oscillations on the brane. Note that these oscillations 
are similar in spirit to the so-called "indirect" four-dimensional neutrino oscillations discussed in Ref. [8], except that here we have an infinite tower of higher-dimensional Kaluza-Klein states mediating our flavor oscillations and we have no explicit mixing angles on the brane.

We shall now discuss several phenomenological features of the "bulk-mediated" flavor oscillations inherent in this model. In general, we are interested in the probabilities $P_{i \rightarrow j}(t)$ that $\left|\nu_{i}\right\rangle$ oscillates into $\left|\nu_{j}\right\rangle$ as a function of time $t$. For $i=j$, this refers to flavor preservation on the brane, while for $i \neq j$ this refers to flavor conversion on the brane. Note that in general, $P_{i \rightarrow j}=P_{j \rightarrow i}$ for all $(i, j)$. For phenomenological purposes, we are mostly interested in the probabilities $P_{1 \rightarrow 1}(t), P_{2 \rightarrow 2}(t)$, and $P_{2 \rightarrow 1}(t)$ where the subscripts $(1,2,3)$ signify $\left(\nu_{e}, \nu_{\mu}, \nu_{\tau}\right)$ respectively. These are ultimately the three probabilities which are relevant for addressing constraints from solar, atmospheric, and long-baseline neutrino experiments.

In the following, we shall rescale all of our mass variables $\left\{m_{i}, m\right\}$ by $R$ so that these variables henceforth correspond to dimensionless quantities. Thus, our model has only four free dimensionless input parameters, $\left\{m, m_{1}, m_{2}, m_{3}\right\}$, while the radius $R$ serves as an overall length scale. These parameters ultimately determine not only the masses of the physical neutrinos, but also their relative effective mixing angles. We shall also define a dimensionless time variable, $\tilde{t} \equiv t /\left(2 p R^{2}\right) \approx\left(1 / 2 R^{2}\right)(L / E)$, where $L \approx c t$ is the spatial distance between the locations of neutrino production and neutrino detection, and $E \approx p c$ is the neutrino energy. We can then plot our probabilities $P_{i \rightarrow j}(t)$ as functions of $\tilde{t}$.

Let us first consider the case when the brane/bulk coupling is extremely small, i.e., $m \ll 1$. In such cases, the mass matrix (4) is nearly diagonal, and there is relatively little mixing between the brane neutrinos and the bulk neutrinos. However, we find that large flavor oscillations can nevertheless be achieved for certain values of the input parameters $\left\{m, m_{i}\right\}$. As an explicit example, let us consider the case $m=0.01, m_{1,2}=1 \mp \delta m / 2$, and $m_{3}=5$, with $\delta m=m_{2}-m_{1}$ free to vary. The resulting neutrino oscillations are shown in Fig. 1. Note that as $\delta m \rightarrow 0$, the resulting flavor oscillation becomes effectively maximal in the sense that we achieve full flavor conversion. In achieving this large flavor oscillation, we are exploiting a resonance between two approximately degenerate brane neutrinos $\left|\nu_{1,2}\right\rangle$ and the first excited Kaluza-Klein bulk neutrino $\left|N^{(1)}\right\rangle$, so that large effective flavor oscillations between the brane neutrinos are mediated indirectly through their oscillations with the bulk neutrino. Nevertheless, no mixing angles on the brane are required.

Even though this flavor mixing appears to resemble a simple two-state oscillation in the limit $\delta m \ll 1$, the time-averaged probabilities for each flavor are not $1 / 2$, but $3 / 8$. This reflects the fact that we are dealing with a three-state oscillation in which $1 / 4$ of the total neutrino probability has been lost to the bulk neutrino $\left|N^{(1)}\right\rangle$, and is hence "sterile" from the point of view of flavor on the brane.

When all three of the brane neutrino flavors are approximately degenerate, so that $m_{1} \approx m_{2} \approx m_{3} \approx k \in \mathbb{Z}$, we find that the bulk neutrino mediates an effective 

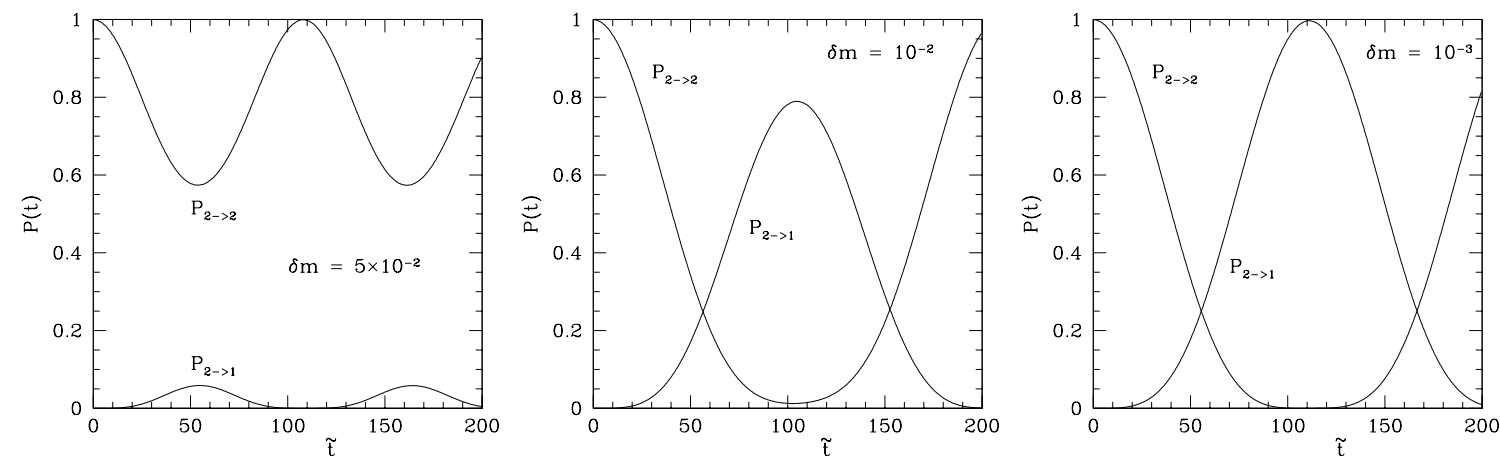

Figure 1: Bulk-mediated neutrino flavor oscillations with $m=0.01$ and $m_{1,2}=1 \mp \delta m / 2$, for various $\delta m$. As discussed in the text, these flavor oscillations are essentially "maximal" even though all mixing angles on the brane are vanishing.

four-state flavor oscillation which closely resembles a three-state oscillation on the brane. As an example, let us consider the case $m=0.01, m_{2}=1$, and $m_{1,3}=$ $1 \mp \delta m / 2$. The resulting probabilities for flavor oscillation and preservation are then shown in Fig. 2. Although the probabilities for flavor preservation and conversion are flavor-independent for short times (top plot of Fig. 2), a strong flavor dependence develops for later times (remaining plots). In fact, the corresponding time-averaged probabilities are given exactly by

$$
\begin{array}{lll}
\overline{P_{1 \rightarrow 1}}=4 / 9, & \overline{P_{1 \rightarrow 2}}=5 / 18, & \overline{P_{1 \rightarrow 3}}=1 / 9, \\
\overline{P_{2 \rightarrow 2}}=5 / 18, & \overline{P_{2 \rightarrow 3}}=5 / 18, & \overline{P_{3 \rightarrow 3}}=4 / 9 .
\end{array}
$$

Thus, if we start with $\left|\nu_{2}\right\rangle$, we see that we are equally likely to have conversion into each flavor, whereas if we start with $\left|\nu_{1}\right\rangle$ or $\left|\nu_{3}\right\rangle$, we are more likely to preserve the initial flavor, with equally spaced declining probabilities for conversion into nearby flavors. However, no matter which flavor of brane neutrino we begin with, we see that exactly $1 / 6$ of the initial probability is ultimately lost into bulk neutrinos.

The above discussion pertains to only several specific choices of parameters $\left\{m, m_{1}, m_{2}, m_{3}\right\}$ in this model; a more complete discussion of these and other scenarios will be presented in Ref. [9]. In all cases, however, we find that we can generate sizable neutrino oscillations even if the brane/bulk coupling parameter $m$ is extremely small and the brane theory is flavor-diagonal. In general, large brane/bulk oscillations are triggered when any of the $m_{i}$ take values that are near integers (in units of $R^{-1}$ ). Note, however, that this does not require a large fine-tuning. When the extra dimensions are large, the corresponding Kaluza-Klein states are relatively closely spaced. Thus, regardless of the specific mass of a flavor neutrino on the brane, there is always a Kaluza-Klein state in the bulk which is effectively degenerate with it. Thus, such brane/bulk resonances are natural and can therefore mediate large brane/bulk neutrino oscillations. On the other hand, when $m$ is small, sizable flavor 

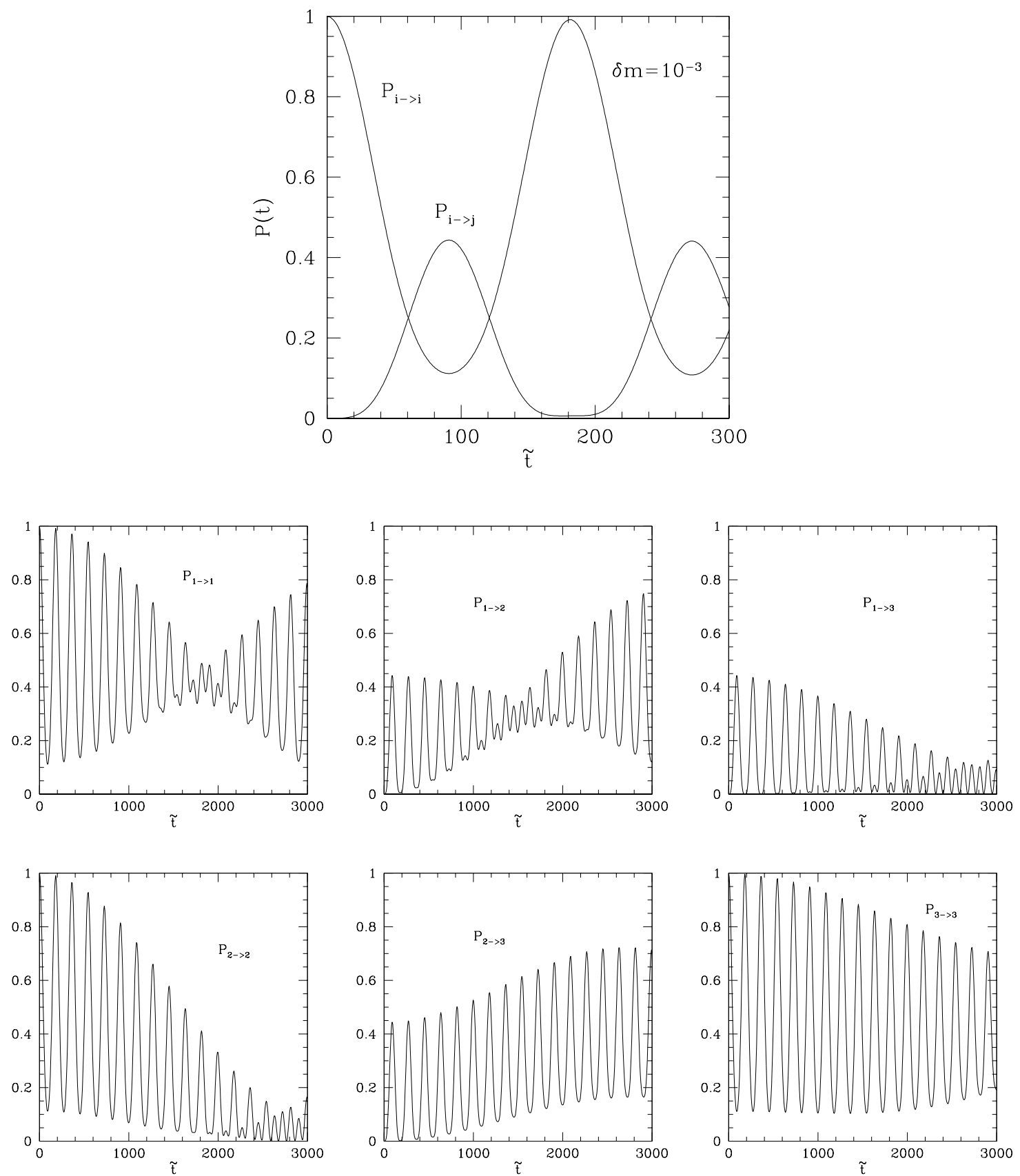

Figure 2: Bulk-mediated neutrino flavor oscillations with $m=0.01, m_{2}=1$, and $m_{1,3}=$ $1 \mp \delta m / 2$ for $\delta m=10^{-3}$. Despite the rather complicated nature of these flavor oscillations, the brane/bulk coupling is small and all flavor mixing angles on the brane are exactly zero. 
oscillations leading to flavor conversion still require approximate flavor degeneracies on the brane.

This situation changes dramatically as the brane/bulk coupling parameter $m$ becomes larger. In such cases, a larger population of bulk Kaluza-Klein neutrino states participates in the neutrino mixings, and it is no longer necessary to have flavor degeneracies on the brane in order to generate sizable bulk-mediated flavor oscillations.

As an example of this, let us consider the case when none of the $m_{i}$ are close to an integer or to each other. For example, we may take $m_{1}=1 / 2, m_{2}=3 / 2$, and $m_{3} \gg 1$. For small values of $m$, this situation gives neither neutrino oscillations nor flavor conversion amongst the two lightest flavors. However, as $m$ increases, both neutrino oscillations and flavor conversions are generated. This is shown in Fig. 3, where we have plotted the time-averaged probabilities for neutrino flavor preservation and conversion as functions of the brane/bulk coupling $m$.

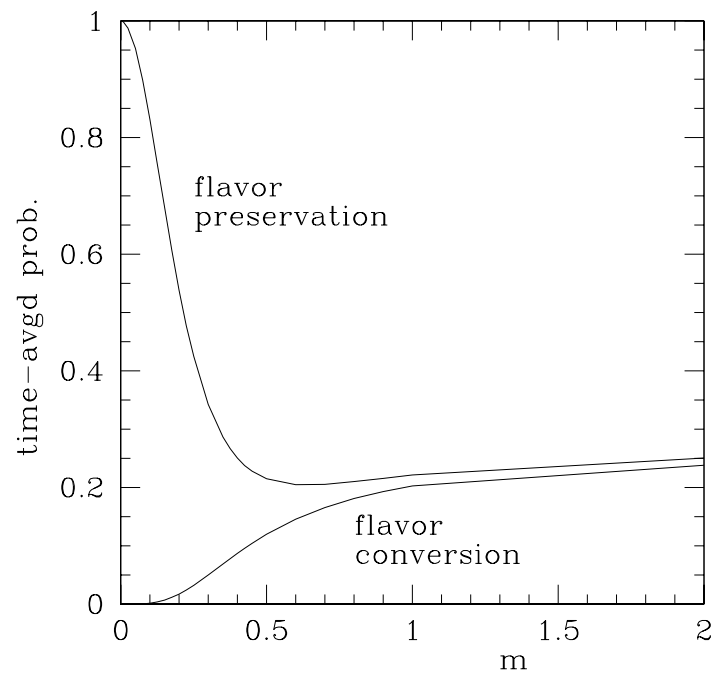

Figure 3: Time-averaged probabilities for bulk-mediated flavor oscillations as functions of the brane/bulk coupling $m$, with $m_{1}=1 / 2, m_{2}=3 / 2$, and $m_{3}=20$. Although the flavor mixing angle on the brane remains zero, significant flavor conversion is generated for relatively small brane/bulk coupling $m$ even without flavor degeneracies on the brane.

These effects can be further enhanced if any of the $m_{i}$ approaches an integer (in units of $R^{-1}$ ), for in such cases there is an additional effect due to a strong resonance oscillation between the corresponding brane neutrino and its degenerate Kaluza-Klein partner in the bulk. This situation mirrors the phenomenon already discussed for the small- $m$ case. As the most extreme case of this, let us consider the situation in which all three of the brane neutrino flavors are approximately degenerate with a bulk neutrino. For concreteness we take $m_{2}=k \in \mathbb{Z}$ and $m_{1,3}=k \mp \delta m / 2$. Choosing $k=1$ and $\delta m=10^{-3}$, we obtain the time-averaged probabilities shown in Fig. 4 . 

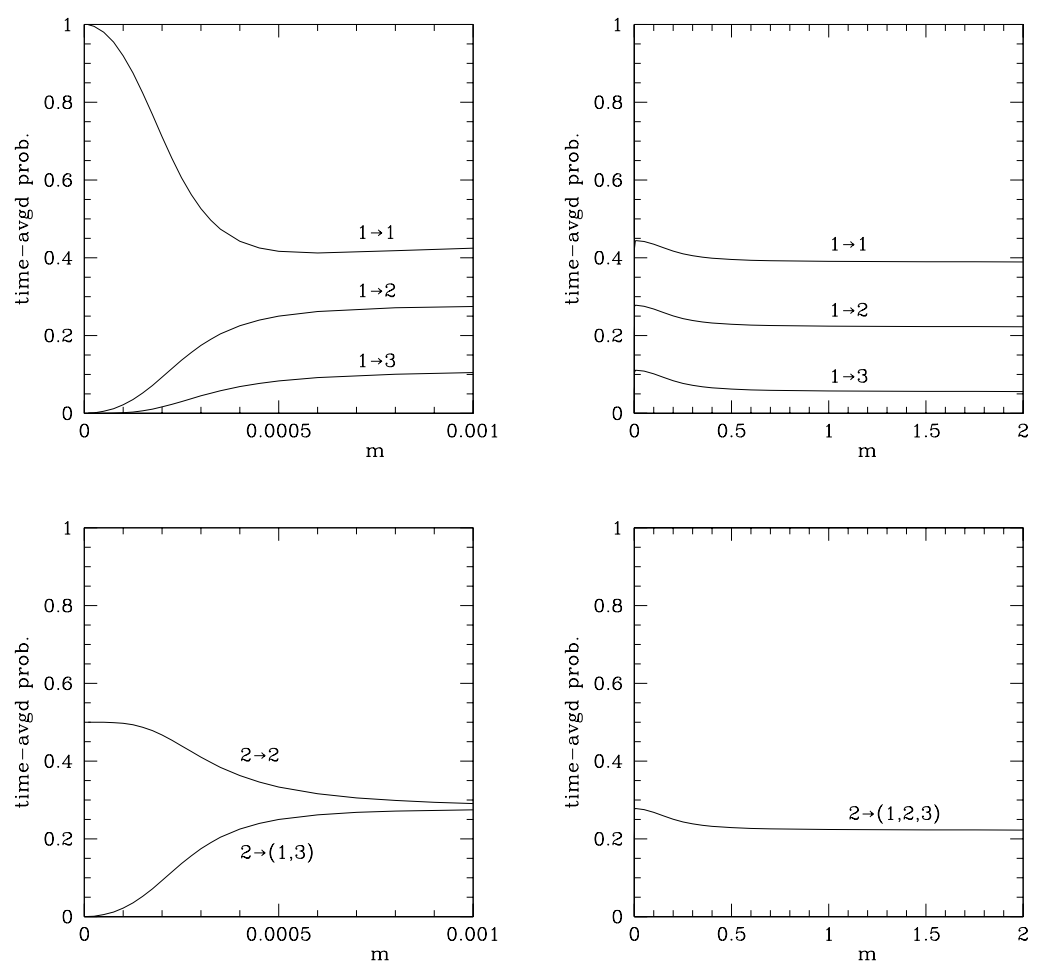

Figure 4: Time-averaged probabilities for bulk-mediated neutrino flavor oscillations, as functions of the brane/bulk coupling $m$. We have taken $m_{2}=1, m_{1,3}=1 \mp \delta m / 2$, and $\delta m=10^{-3}$. Note that with these parameters, $\overline{P_{3 \rightarrow 3}}=\overline{P_{1 \rightarrow 1}}$ for all $m$.

This figure may be understood as follows. As $m \rightarrow 0$, we expect that the preservation probabilities should go to 1 while the conversion probabilities should go to zero. This is indeed what happens, except that as $m \rightarrow 0$, the time-averaged probability $\overline{P_{2 \rightarrow 2}}$ goes not to 1 but to $1 / 2$. This reflects the fact that for any non-zero value of $m$, there continues to exist a maximal two-state resonance between $\left|\nu_{2}\right\rangle$ and $\left|N^{(1)}\right\rangle$.

As $m$ increases into the range $5 \times 10^{-4} \lesssim m \lesssim 0.1$, we see from Fig. 1 that the neutrino probabilities then settle into an effective four-state resonance oscillation pattern for which the time-averaged probabilities are given in (7). As we see from Fig. 1, these time-averaged resonance values persist over several orders of magnitude in $m$. Finally, as $m$ increases beyond 0.1, larger numbers of Kaluza-Klein states begin to participate in the neutrino oscillations. This increases the probabilities for oscillations into bulk neutrinos, which in turn causes our time-averaged neutrino probabilities on the brane to decline relative to their values in (7). Remarkably, however, we see from Fig. 1 that even though these probabilities decline from the values given in (7), they still preserve their relative spacings as functions of $m$. Moreover, we see that the time-averaged probabilities on the brane do not fall to zero as $m \rightarrow \infty$, but instead 
take the non-zero asymptotic values

$$
\begin{array}{rlrl}
\overline{P_{1 \rightarrow 1}}=7 / 18, & \overline{P_{1 \rightarrow 2}}=2 / 9, & \overline{P_{1 \rightarrow 3}}=1 / 18, \\
\overline{P_{2 \rightarrow 2}}=2 / 9, & \overline{P_{2 \rightarrow 3}}=2 / 9, & & \overline{P_{3 \rightarrow 3}}=7 / 18 .
\end{array}
$$

The fact that these asymptotic probabilities are non-zero illustrates that even when an infinite number of bulk Kaluza-Klein states contribute to the neutrino oscillations, and even when the brane/bulk coupling is infinite, we still do not lose all of our initial neutrino probability into the bulk states! Instead, as $m \rightarrow \infty$, we lose only $1 / 3$ of the initial probability into bulk neutrinos. In other words, we see that $2 / 3$ of the original probability remains "on the brane" even when $m \rightarrow \infty$. This is in fact a completely general feature of our model, and holds regardless of the specific values of the $m_{i}$. Moreover, this property generalizes: with $n_{f}$ flavors on the brane, we find that only $1 / n_{f}$ of the initial neutrino probability is lost into bulk neutinos.

This surprising observation suggests that in our model, the large- $m$ case may be able to evade various four-dimensional bounds (such as those from supernova cooling rates) which ordinarily restrict the sizes of mixings with sterile neutrinos. Indeed, our results such as those in Eq. (8) apply for large values of $m$ for which it is not possible to employ constraints derived using a "perturbative" approach in which only the lightest few Kaluza-Klein bulk neutrinos mix significantly. Rather, an exact treatment is required in which the full Kaluza-Klein tower is incorporated simultaneously, for in our model it is the entire Kaluza-Klein tower that plays a role in reconverting bulk neutrinos back into neutrino flavors on the brane as the brane/bulk coupling becomes large. This issue, especially in conjunction with MSW matter effects, will be discussed further in Ref. [9].

Thus, to summarize, we see that it is possible to have large effective "bulkmediated" flavor mixing on the brane even when all flavor mixing angles on the brane are zero. Moreover, this occurs even when the bulk theory is flavor-neutral and the brane/bulk couplings are flavor-blind. This illustrates that in higher dimensions, it may not be necessary to have large flavor mixing angles on the brane in order to accommodate experimental data; small (or even vanishing) flavor mixing angles on the brane may suffice. Furthermore, even though this model contains only five free parameters, it yields a surprisingly rich neutrino oscillation phenomenology, and exploits the higher-dimensional nature of the bulk in an essential way. Moreover, its essential structure involving only one bulk neutrino leads to a radically different neutrino phenomenology for large brane/bulk coupling than is possible in models with multiple bulk neutrinos, and suggests that this limit might actually evade supernova cooling constraints. Thus, this model might be profitably used as the basis

* Note that this property relies crucially on the fact that we have taken only one bulk neutrino in our model; in other words, we have not extended flavor into the bulk. By contrast, if we had taken a separate bulk neutrino for each brane neutrino, we would have instead found that all neutrino probability is ultimately lost into sterile bulk neutrinos as $m \rightarrow \infty$. 
of a detailed investigation of the experimental viability of various higher-dimensional neutrino oscillation mechanisms. This will be discussed further in Ref. [9].

\section{Acknowledgments}

We wish to thank C.P. Burgess, B. Campbell, L. Dixon, Y. Grossman, S. Lola, R. Mohapatra, K. Orginos, and M.H. Reno for discussions. This work was supported in part by the National Science Foundation under Grant PHY-0071054 and by the Department of Energy under Grants DE-FG02-95ER40906 and DE-FG03-93ER40792. We also wish to acknowledge the hospitality of the Aspen Center for Physics and the CERN Theory Division where portions of this work were completed.

\section{References}

[1] Y. Suzuki, SuperK Collaboration, talk at Neutrino 2000: XIX ${ }^{\text {th }}$ International Conference on Neutrino Physics and Astrophysics, Sudbury, Canada, June 2000.

[2] H. Sobel, SuperK Collaboration, talk at Neutrino 2000: XIX ${ }^{\text {th }}$ International Conference on Neutrino Physics and Astrophysics, Sudbury, Canada, June 2000.

[3] G. Mills, LSND Collaboration, talk at Neutrino 2000: XIX ${ }^{\text {th }}$ International Conference on Neutrino Physics and Astrophysics, Sudbury, Canada, June 2000.

[4] K.R. Dienes, E. Dudas, and T. Gherghetta, Nucl. Phys. B557 (1999) 25 hep-ph/9811428.

[5] N. Arkani-Hamed et al., hep-ph/9811448.

[6] See, e.g., I. Antoniadis, Phys. Lett. B246 (1990) 377; E. Witten, Nucl. Phys. B471 (1996) 135; J.D. Lykken, Phys. Rev. D54 (1996) 3693; N. Arkani-Hamed, S. Dimopoulos, and G. Dvali, hep-ph/9803315; hep-ph/9807344; I. Antoniadis, N. Arkani-Hamed, S. Dimopoulos, and G. Dvali, hep-ph/9804398; K.R. Dienes, E. Dudas, and T. Gherghetta, Phys. Lett. B436 (1998) 55 hep-ph/9803466]; Nucl. Phys. B537 (1999) 47 [hep-ph/9806292]; hep-ph/9807522; G. Shiu and S.-H.H. Tye, hep-th/9805157; Z. Kakushadze and S.-H.H. Tye, hep-th/9809147.

[7] See, e.g., G. Dvali and A. Smirnov, hep-ph/9904211; A. Pilaftsis, hep-ph/9906228; R.N. Mohapatra, S. Nandi, and A. Perez-Lorenzana, hep-ph/9907520; A. Das and O. Kong, hep-ph/9907272; A. Ioannisian and A. Pilaftsis, hep-ph/9907522; G.C. McLaughlin and J.N. Ng, hep-ph/9909558; nucl-th/0003023; A.E. Faraggi and M. Pospelov, hep-ph/9901299; R.N. Mohapatra and A. Perez-Lorenzana, hep-ph/9910474; hep-ph/0006190; A. Ioannisian and J.W.F. Valle, hep-ph/9911349; R. Barbieri, P. Creminelli, and 
A. Strumia, hep-ph/0002199; K.R. Dienes, hep-ph/0004129; K. Agashe, N.G. Deshpande, and G.H. Wu, hep-ph/0006122; E. Ma, G. Rajasekaran, and U. Sarkar, hep-ph/0006340; A. Lukas et al., hep-ph/0008049.

[8] B.W. Lee et al., Phys. Rev. Lett. 38 (1977) 937; S.B. Treiman, F. Wilczek, and A. Zee, Phys. Rev. D16 (1977) 152; K.S. Babu, J.C. Pati, and F. Wilczek, Phys. Lett. B359 (1995) 351.

[9] K.R. Dienes and I. Sarcevic, to appear. 